\title{
An entrainment model for lazy turbulent plumes
}

\author{
P. Carlotti ${ }^{1} \dagger$ and G.R. Hunt ${ }^{2}$ \\ ${ }^{1}$ Laboratoire Central de la Préfecture de Police, 39bis rue de Dantzig, 75015 Paris, France. \\ ${ }^{2}$ Department of Engineering, University of Cambridge, Trumpington Street, \\ Cambridge CB2 1PZ, UK.
}

(Received 25 October 2016)

An entrainment model for lazy turbulent plumes is proposed, the resulting solutions of the plume conservation equations are developed and the implications for plume behaviour are considered and compared with the available experimental data. Indeed the applicability of the classic solutions of the conservation equations subject to source conditions that produce lazy plumes, i.e. those with suitably high source Richardson number, contain an essential weakness: the underlying assumption of a constant entrainment coefficient. Whilst entrainment models prescribing the dependence of the entrainment coefficient with local Richardson number have been proposed for forced plumes, corresponding formulations for lazy plumes have not until now been considered. In the context of saline plumes, the model is applied directly. For hot gaseous plumes, we use a modified definition of buoyancy flux to recover a constant buoyancy flux in a non-stratified environment, despite specific heat varying with temperature, as shown in the appendix. After a rapid review of existing forced plume formulations of entrainment, a power-law variation is adopted for the lazy plume. The plume equations are solved for the parameter $0 \leq \omega<1$, where $\omega$ denotes the exponent of the power law. The cases of pure plumes and lazy plumes are then analysed in more detail; to our knowledge this represents the first modelling of variable entrainment for lazy plumes. Specifically, it is shown that classic plume theory is recovered for $\omega=0$, while for $\omega=1 / 5$ the plume equations may be solved using usual functions (notably polynomials) only. The results of the models for these cases are very similar, which advocates the idea of selecting systematically $\omega=1 / 5$, instead of $\omega=0$, for cases where the effect of variation of entrainment is weak, since the new model leads to simple calculations. In the case of very lazy plumes it is shown that, provided a relevant value of $\omega$ is chosen, the new model reproduces well the available experimental results.

\section{Introduction}

Questions concerning the development of flows above isolated sources of buoyancy may be approached either with the aim of computing full velocity and density fields, thereby requiring solution of the Navier-Stokes equations, or with the aim of computing a limited number of parameters only and as functions of height. The latter approach is exemplified in the seminal work of Morton et al. (1956) (hereafter MTT). They concentrate on time-averaged fluxes through horizontal planes. They work in the Boussinesq case (small density variations), and use buoyancy flux $B(z)$, volume flux $Q(z)$ and momentum flux $M(z)$, where $z$ denotes the height above the physical source at $z=0$. In order to generalise to large density variations, Carlotti \& Hunt (2005) showed that it is more straightforward

$\dagger$ Email address for correspondence: Pierre.Carlotti@interieur.gouv.fr 
to use mass flux $G(z)$ than volume flux, leading to the following definition of the fluxes ( $\rho_{a}$ denoting the ambient density, which is assumed to be uniform in the present paper)

$$
\begin{aligned}
B(z) & =\iint g \frac{\rho_{a}-\rho}{\rho_{a}} W d S \\
M(z) & =\iint \rho W^{2} d S \\
G(z) & =\iint \rho W d S,
\end{aligned}
$$

where $W$ denotes the vertical velocity of the plume and integration is over horizontal planes.

Regarding notation, we adopt the following throughout: subscript $a$ refers to the ambient; subscript 0 to the reference cases of uniform entrainment or of a pure plume (which reduces to uniform entrainment with our model); subscript $T$ denotes 'top-hat' profile; subscript $s$ denotes the quantities at the source in the theory, i.e. the lowest possible height of existence of the plume considered; and subscript $n$ refers to quantities at the exit plane of the plume nozzle in experiments.

From these fluxes, MTT define the so-called top-hat radius $b_{T}$, vertical velocity $W_{T}$ and density $\rho_{T}$ from

$$
B(z)=\pi g \frac{\rho_{a}-\rho_{T}}{\rho_{a}} W_{T} b_{T}^{2} \quad M(z)=\pi \rho_{T} W_{T}^{2} b_{T}^{2} \quad G(z)=\pi \rho_{T} W_{T} b_{T}^{2} .
$$

Mass, momentum and enthalpy conservation then lead to the following ordinary differential equations for an unstratified ambient fluid (MTT actually used a slightly different formulation, but with the same assumptions):

$$
\begin{aligned}
\frac{d G}{d z} & =2 \pi \rho_{a} b_{T} u_{e}(z) \\
\frac{d M}{d z} & =\pi b_{T}^{2}\left(\rho_{a}-\rho_{T}\right) g \\
\frac{d B}{d z} & =0 .
\end{aligned}
$$

The first equation carries the main insight of the MTT paper: the plume is conjectured to entrain ambient fluid with an entrainment speed $u_{e}(z)$ at the plume perimeter. MTT further assume that the entrainment velocity is proportional to the vertical velocity, $u_{e}(z)=\alpha W_{T}(z)$, with $\alpha$ the entrainment coefficient (these ideas were already germinating in G.I. Taylor's work during World War II, see Taylor (1958), and independently, pre-war, by Zeldovich (1937)). The latter assumption leads to a full set of closed equations for which self-similar solutions may be found (for the so-called pure plume), together with more complicated solutions for forced plumes (Morton 1959) or lazy plumes (Hunt $\&$ Kaye 2001) depending on the value of the plume Richardson number $\Gamma$ :

$$
\Gamma=\rho_{a}^{1 / 2} \frac{B G^{2}}{M^{5 / 2}}
$$

The MTT theory is applicable to Boussinesq plumes in which density contrast is small (i.e. $\left|\rho_{a}-\rho_{T}\right| \ll \rho_{a}$ ). This theory was generalised to non-Boussinesq plumes by Rooney $\&$ Linden (1996) by introducing a factor $\sqrt{\frac{\rho_{T}}{\rho_{a}}}$, assuming a closure of the form

$$
u_{e}(z)=\alpha_{0} W_{T}(z) \sqrt{\frac{\rho_{T}}{\rho_{a}}} .
$$


The subscript 0 on $\alpha$ is introduced here to indicate that its value is assumed to be independent of other plume quantities, being kept equal to its pure-plume value.

With the present notation, the pure-plume self-similar solution of Rooney \& Linden (1996) leads to a constant value of the Richardson number $\Gamma=\Gamma_{0}$, with

$$
\Gamma_{0}=\frac{8}{5} \sqrt{\pi} \alpha_{0}
$$

Carlotti \& Hunt (2005) derived a general (lazy and forced) solution based on the assumptions of Rooney \& Linden (1996). This general solution was further explored and discussed by Hunt \& van den Bremer (2010), and Candelier \& Vauquelin (2012), who expressed it in a simpler way. However, these solutions still contain two weaknesses with regards to their applicability in the prediction of very hot gas plumes rising in a cooler environment: they assume constant specific heat of the gas and a constant entrainment coefficient. Addressing the latter weakness is our primary focus herein.

The paper is organised as follows. $\S 2$ discusses formulations of entrainment dependent on the local plume Richardson number and proposes a power-law formulation. This is applied to lazy plumes in $\S 3$, and $\S 4$ considers the specific case of a lazy plume issuing from a nozzle, allowing comparison of the theory presented in $\S 2$ and $\S 3$ with the experimental results of Kaye \& Hunt (2009). In $\S 5$ we present our conclusions. To address the second weakness, Appendix A focusses on an alternative definition of buoyancy flux for hot gaseous plumes with variable specific heat which allows one to apply the theory presented in the present paper to this context, namely, where the traditional buoyancy flux is not constant.

\section{Variable entrainment plume models}

\subsection{Basic set of equations and variable entrainment}

In the following, we work only with the flux variables $(B, M, G)$ and use algebra to recast the plume conservation equations in this context, leading to

$$
\begin{aligned}
\frac{d B}{d z} & =0 \\
\frac{d M}{d z} & =\rho_{a} \frac{B G}{M} \\
\frac{d G}{d z} & =2 \sqrt{\pi} \sqrt{\rho_{a} M} \cdot \alpha .
\end{aligned}
$$

We assume that entrainment depends on local plume quantities $\left(B, M, G, \rho_{T}, \rho_{a}\right)$ only. From dimensional analysis we find that $\alpha=\alpha\left(\Gamma, \rho_{T} / \rho_{a}\right)$.

Using (2.3) allows us to compute $\alpha$ solely from the knowledge of fluxes:

$$
\alpha=\frac{1}{2 \sqrt{\pi \rho_{a} M}} \frac{d G}{d z} .
$$

Using this equation, Pham et al. (2005) measured entrainment as a function of height in a nearly Boussinesq plume (their figure 16) and showed that, close to the source, where their plume is very lazy, entrainment is much increased. This measurement is also confirmed by the relatively low resolution LES of Lamalle et al. (2013). For lazy plume sources, where the near-field behaviour is of primary interest, the classic MTT approach to plume modelling has been applied previously with some success (e.g. Fannelop \& Weber (2003), Hunt \& Kaye (2005), Candelier \& Vauquelin (2012)) despite the underlying modelling assumptions, e.g. the morphology being long and thin in the near field and 
the pressure being hydrostatic, not strictly applying. Wang \& Law (2002) review the literature covering models for turbulent entrainment (mainly Priestley \& Ball (1955) and List (1982)), and suggest the following two formulations primarily designed for forced and pure plumes:

$$
\begin{aligned}
& \alpha=\alpha_{j e t}+\left(\alpha_{0}-\alpha_{j e t}\right) \frac{\Gamma}{\Gamma_{0}} \\
& \alpha=\alpha_{j e t}\left(\frac{\alpha_{0}}{\alpha_{j e t}}\right)^{\Gamma / \Gamma_{0}} .
\end{aligned}
$$

Here, $\alpha_{0}$ is the entrainment constant in a pure plume and $\alpha_{j e t}$ is the entrainment constant in a jet. Besides reducing to the entrainment coefficient for a pure jet and a pure plume in the limits as $\Gamma \rightarrow 0$ and as $\Gamma \rightarrow \Gamma_{0}$, the physical justification for the variation with local Richardson number for forced plumes was somewhat limited for both models when first posed. That said, experimental measurements on thermal plumes (e.g. by Ezzamel et al. (2015)) and predictions of direct numerical simulations (e.g. by van Reeuwijk et al. (2016)) support the use of these models within classic plume theory. Note that these formulations lead to unrealistic values of entrainment coefficient for very lazy plumes $\Gamma \gg 1$, see Kaye \& Hunt (2009) who use values of $\Gamma / \Gamma_{0}$ of order up to $10^{7}$.

Wang \& Law (2002) use Gaussian profiles and follow List (1982) for the values of $\alpha_{\text {jet }}$ and $\alpha_{0}$, taking $\alpha_{j e t}=0.0535$ and $\alpha_{0}=0.0833$. With top-hat profiles, as in the present paper, the corresponding values are $\alpha_{\text {jet }}=0.0757$ and $\alpha_{0}=0.118$.

In the present work, we focus on pure and lazy plumes and consider the power-law formulation (with $0 \leq \omega<1$, as we will see later):

$$
\alpha=\alpha_{0}\left(\frac{\Gamma}{\Gamma_{0}}\right)^{\omega} .
$$

The reasons for this proposed form of entrainment function are essentially three-fold. First, consistent at least qualitatively with measurement (e.g. Pham et al. (2005), Ezzammel et al. (2015)), this form results in enhanced entrainment in lazy plumes compared with pure plumes, while being robust for very large values of $\Gamma / \Gamma_{0}$. Second, the natural scalings that arise from the application of plume theory with an entrainment model as per (2.7) enable the near-field measurements of mass flow rate made in lazy plumes to be collapsed when scaled (see §4) and the predictions show good agreement with the available data. A survey of the open literature reveals that there is limited experimental data available on the near-field behaviour of highly lazy plumes, with measurements pertaining to entrainment restricted to those on saline Boussinesq plumes in freshwater environments made by Kaye \& Hunt (2009). Surprisingly, there appears to be a complete absence of experimental data on the near-field entrainment behaviour of highly-lazy non-Boussinesq plumes with the exception of Pham et al., for which the values of buoyancy and flow rates are not documented. We should also stress that this formulation is consistent with energy constraints and entrainment formulations proposed by van Reeuwijk and Craske (2015). Finally, with a suitable choice of $\omega$, our formulation for the entrainment coefficient and that of Priestley \& Ball (1955) (2.5) are very close for $\Gamma \approx \Gamma_{0}$. In other words, (2.7) not only reduces to the widely accepted pure-plume behaviour as $\Gamma \rightarrow \Gamma_{0}$, but also is very close for weakly lazy plumes, $\Gamma \gtrsim \Gamma_{0}$, as now shown. The three expressions for variable entrainment, (2.5)-(2.7), have the following gradients at $\Gamma=\Gamma_{0}$, respectively

$$
\alpha_{0}-\alpha_{j e t}, \quad-\alpha_{0} \ln \left(1-\frac{\alpha_{0}-\alpha_{j e t}}{\alpha_{0}}\right), \quad \omega \alpha_{0} .
$$

Therefore, if we let $\omega=\left(\alpha_{0}-\alpha_{j e t}\right) / \alpha_{0}$, the Priestley \& Ball (1955) formulation (2.5) 
and our proposed power-law formulation (2.7) are very close for $\Gamma \approx \Gamma_{0}$. Further, if we have $\frac{\alpha_{0}-\alpha_{j e t}}{\alpha_{0}} \ll 1$, all three formulations are very close for $\Gamma \approx \Gamma_{0}$. Note that if we take, following Wang \& Law (2002), $\alpha_{\text {jet }}=0.0757$ and $\alpha_{0}=0.118$, this leads us to assume $\omega \approx 0.36$.

For lazy plumes, $\Gamma>\Gamma_{0}$, our formulation for $\alpha,(2.7)$, consistent with measurements, yields entrainment coefficients that exceed the pure-plume value $\alpha_{0}$. For pure plumes, $\Gamma=\Gamma_{0}$, (2.7) reduces to $\alpha=\alpha_{0}$, as do the Priestley \& Ball (1955) and List (1982) formulations. However, for $\Gamma \ll \Gamma_{0},(2.7)$ does not recover $\alpha=\alpha_{j e t}$ and, as such, we will not consider further our model for these highly forced plumes.

\subsection{Plume invariant}

Combining (2.3) and (2.2), with entrainment given by (2.7), one finds

$$
\frac{d M}{d G}=\frac{\sqrt{\rho_{a}}}{2 \sqrt{\pi}} \cdot \frac{1}{\alpha_{0}}\left(\frac{\Gamma}{\Gamma_{0}}\right)^{-\omega} \cdot \frac{B G}{M^{3 / 2}} .
$$

Some algebra leads to

$$
\frac{d M}{d G}=\frac{4}{5}\left(\frac{\sqrt{\rho_{a}} B}{\Gamma_{0}}\right)^{1-\omega} \cdot \frac{G^{1-2 \omega}}{M^{3 / 2-5 \omega / 2}}
$$

The above equation may be integrated and we find that, for $0 \leq \omega<1$, the quantity

$$
J=\frac{1}{2}\left(G^{2(1-\omega)}-\left(\frac{\Gamma_{0}}{\sqrt{\rho_{a}} B}\right)^{1-\omega} M^{5(1-\omega) / 2}\right)
$$

is independent of height $z$ in the plume and, thereby, provides us with a plume invariant (the factor of $\frac{1}{2}$ is included for simplicity in the next section). The constant $J$ may be computed from its value at the source (recalling that the subscript $(\cdot)_{s}$ refers to the source and $(\cdot)_{0}$ to the pure-plume reference case):

$$
J=J_{s}=\frac{1}{2}\left(G_{s}^{2(1-\omega)}-\left(\frac{\Gamma_{0}}{\sqrt{\rho_{a}} B}\right)^{1-\omega} M_{s}^{5(1-\omega) / 2}\right) .
$$

This is a generalisation of the concept first presented in Carlotti \& Hunt (2005) (therein constant entrainment was assumed, i.e. $\omega=0$ ), where for the present notation, the invariant $J_{\omega=0}=\frac{1}{2}\left[G^{2}-\left(\Gamma_{0} /\left(\sqrt{\rho_{a}} B\right)\right) M^{5 / 2}\right]$ was used as an intermediate step to solve the general (pure, lazy and forced) constant entrainment plume equations.

\subsection{Case $\omega=1$}

The above formulation of entrainment does not hold when $\omega=1$ since $(2.10)$ becomes

$$
\frac{d M}{d G}=\frac{4}{5} \frac{M}{G},
$$

which may not be integrated with power laws but instead with logarithms, leading to

$$
\frac{G^{4}}{M^{5}}=\text { const. }=\frac{G_{s}^{4}}{M_{s}^{5}}
$$

As a consequence, in such a plume, we would have $\Gamma=\Gamma_{s}$ independent of height; this is contradictory to all experimental evidence which shows that $\Gamma(z)$ converges towards the pure-plume value with height (e.g. Ezzamel et al. 2015), regardless of the source value $\Gamma_{s}$. For this reason, we will not consider any further the case $\omega=1$. 


\subsection{Pure plume $J=0$}

When $J=0,(2.11)$ leads directly to $G^{2}=\left[\Gamma_{0} /\left(\sqrt{\rho_{a}} B\right)\right] M^{5 / 2}$ for any value of $\omega \in[0,1)$. Therefore $\Gamma=\Gamma_{0}$ at all heights, showing that this case corresponds to a pure plume. Adopting the expression for $G^{2}$ into (2.2), we get

$$
\frac{d M}{d z}=\rho_{a}^{3 / 4} B^{1 / 2} \Gamma_{0}^{1 / 2} M^{1 / 4}
$$

leading to the classic non-Boussinesq point source solution (Rooney \& Linden (1996) and Carlotti \& Hunt (2005)) on taking $M_{s}=0$ and $G_{s}=0$ at the source:

$$
\begin{aligned}
G & =\left(\frac{3}{4}\right)^{5 / 3} \Gamma_{0}^{4 / 3} \rho_{a} B^{1 / 3} z^{5 / 3} \\
M & =\left(\frac{3}{4}\right)^{4 / 3} \Gamma_{0}^{2 / 3} \rho_{a} B^{2 / 3} z^{4 / 3} .
\end{aligned}
$$

\section{Lazy plumes $J>0$}

\subsection{General plume equations}

Together with $J$, we introduce

$$
I=\frac{1}{2}\left(G^{2(1-\omega)}+\left(\frac{\Gamma_{0}}{\sqrt{\rho_{a}} B}\right)^{1-\omega} M^{5(1-\omega) / 2}\right) .
$$

As a consequence of this definition

$$
\begin{aligned}
G^{2(1-\omega)} & =I+J \\
\left(\frac{\Gamma_{0}}{\sqrt{\rho_{a}} B}\right)^{1-\omega} M^{5(1-\omega) / 2} & =I-J .
\end{aligned}
$$

Differentiating (3.2) and using (2.3), some algebra shows that

$$
\frac{d I}{d z}=\frac{5}{2}(1-\omega) \Gamma_{0}^{4 / 5} \rho_{a}^{3 / 5} B^{1 / 5} \cdot(I+J)^{\frac{1}{2-2 \omega}}(I-J)^{\frac{1-5 \omega}{5-5 \omega}} .
$$

Equation (3.4), together with the fact that $J$ is independent of height, gives the complete set of equations for the plume in the $J>0$ case. Evidently, the solution for the plume will depend on the value of $J$. Note that this set of equations is also valid when $J=0$, even though we did not need to use it because there was a simpler method.

\subsection{Non-dimensional form of the I-equation and acceleration length}

Since $J \neq 0$ for a lazy plume, we may define $\mathcal{I}=I / J$. Note that $\mathcal{I} \geq 1$ for any value of height. The case $\mathcal{I}=1$ corresponds to $I=J$, this is $M=0$. Since $M$ increases with height, this corresponds to the lowest possible height of existence of the lazy plume. In the present section, we will therefore consider this height as the origin of heights and as the source of the plume. Since $M_{s}=0$ at this height, we also have with $G_{s}$ the value of $G$ at the source,

$$
J=\frac{1}{2} G_{s}^{2(1-\omega)}
$$

Non-dimensionalising, (3.4) reduces to

$$
\frac{d \mathcal{I}}{d \mathcal{Z}}=(\mathcal{I}+1)^{\frac{1}{2-2 \omega}}(\mathcal{I}-1)^{\frac{1-5 \omega}{5-5 \omega}},
$$


with $\mathcal{Z}=z / L$, and

$$
L=\frac{2}{5 \Gamma_{0}^{4 / 5}(1-\omega)} \frac{J^{\frac{3}{10(1-\omega)}}}{\rho_{a}^{3 / 5} B^{1 / 5}}=\frac{2^{\frac{7}{10}}}{5 \Gamma_{0}^{4 / 5}} \frac{2^{\frac{-3 \omega}{10(1-\omega)}}}{1-\omega} \frac{G_{s}^{3 / 5}}{\rho_{a}^{3 / 5} B^{1 / 5}} .
$$

The quantity $L$ is a modified formulation of the plume acceleration length scale (e.g. Fischer et al. 1979, Caulfield 1991, Kaye 2008), and represents the characteristic vertical scale over which the very lazy plume accelerates to approach its large-height asymptotic pure-plume state.

Writing $L_{0}$ as the acceleration length scale for a constant entrainment plume $\omega=0$, we have

$$
\frac{L}{L_{0}}=\frac{2^{\frac{-3 \omega}{10(1-\omega)}}}{1-\omega} .
$$

This ratio increases from $L / L_{0}=1$ to 2.2 as $\omega$ increases from $\omega=0$ to 0.8 , and then decreases rapidly to reach the value $L / L_{0}=0$ as $\omega \rightarrow 1$. This change of behaviour is to be analysed bearing in mind that $\omega=1$ is physical, as explained before, and values near $\omega=1$ are unlikely to be realistic.

\subsection{Integration of the I-equation}

For general $\omega$, the ordinary differential equation (3.6), although first order, does not permit a solution in terms of usual functions, and we define

$$
\hat{\Phi}_{\omega}(X)=\int_{1}^{X} \frac{d u}{(u+1)^{\frac{1}{2-2 \omega}}(u-1)^{\frac{1-5 \omega}{5-5 \omega}}} .
$$

With the choice of origin of height previously discussed, (3.6) leads to $\hat{\Phi}_{\omega}(\mathcal{I})-\hat{\Phi}_{\omega}(1)=\mathcal{Z}$. Since $\hat{\Phi}_{\omega}(1)=0$, we get

$$
\hat{\Phi}_{\omega}(\mathcal{I})=\mathcal{Z}
$$

This is also, with $\Phi_{\omega}$ being defined as the inverse function of $\hat{\Phi}_{\omega}$,

$$
\mathcal{I}=\Phi_{\omega}(\mathcal{Z})
$$

From (3.2) and (3.3), we may now write

$$
\begin{aligned}
G & =G_{s} \cdot\left(\frac{\Phi_{\omega}(\mathcal{Z})+1}{2}\right)^{1 /(2(1-\omega))} \\
M & =\left(\frac{\sqrt{\rho_{a}} B}{\Gamma_{0}}\right)^{2 / 5} G_{s}^{4 / 5} \cdot\left(\frac{\Phi_{\omega}(\mathcal{Z})-1}{2}\right)^{2 /(5(1-\omega))} .
\end{aligned}
$$

This is the solution of the plume equations in the lazy case for the variable entrainment considered herein. Defining the top-hat density ratio at the source as

$$
\eta_{s}=\frac{\rho_{T}(\mathcal{Z}=0)}{\rho_{a}}
$$

we may use (1.4) to express $G_{s}$ as

$$
G_{s}=\frac{\rho_{a} B}{g} \frac{\eta_{s}}{1-\eta_{s}}
$$




\subsection{Pure-plume asymptotics for large height}

For large $\mathcal{Z}$, using classic theorems for the asymptotics of integrals depending on their bounds, $\Phi_{\omega}(\mathcal{Z}) \sim\{3 \mathcal{Z} /[10(1-\omega)]\}^{10(1-\omega) / 3}$ and therefore

$$
\begin{aligned}
G & \sim\left(\frac{3}{10(1-\omega)}\right)^{5 / 3} \frac{G_{s}}{2^{1 /(2(1-\omega))}} \mathcal{Z}^{5 / 3} \\
M & \sim\left(\frac{3}{10(1-\omega)}\right)^{4 / 3}\left(\frac{\sqrt{\rho_{a}} B}{\Gamma_{0}}\right)^{2 / 5} \frac{G_{s}^{4 / 5}}{2^{2 /(5(1-\omega))}} \mathcal{Z}^{4 / 3} .
\end{aligned}
$$

After some algebra, this reduces to

$$
\begin{aligned}
G & \sim\left(\frac{3}{4}\right)^{5 / 3} \Gamma_{0}^{4 / 3} \rho_{a} B^{1 / 3} z^{5 / 3} \\
M & \sim\left(\frac{3}{4}\right)^{4 / 3} \Gamma_{0}^{2 / 3} \rho_{a} B^{2 / 3} z^{4 / 3} .
\end{aligned}
$$

Thus, whatever the value of $\omega$, asymptotically for large $z$, pure-plume behaviour (see (2.16) and $(2.17))$ is recovered.

\subsection{Lazy plumes with $\omega=1 / 5$}

The case $\omega=1 / 5$ is interesting because it is simple to solve for as (3.9) may be integrated directly and the inverse $\Phi_{1 / 5}$ expressed in terms of usual functions, thus $\Phi_{1 / 5}(\mathcal{Z})=$ $\left(3 \mathcal{Z} / 8+2^{3 / 8}\right)^{8 / 3}-1$. Equations (3.12) and (3.13) for $G$ and $M$ become

$$
\begin{aligned}
& G=G_{s} \cdot\left(1+\frac{3}{8 \cdot 2^{3 / 8}} \mathcal{Z}\right)^{5 / 3} \\
& M=\left(\frac{\sqrt{\rho_{a}} B G_{s}^{2}}{\Gamma_{0}}\right)^{2 / 5}\left(\frac{8}{3}\right)^{4 / 3} \cdot\left[\frac{1}{2}\left(\frac{3}{8} \mathcal{Z}+2^{3 / 8}\right)^{8 / 3}-1\right]^{1 / 2} .
\end{aligned}
$$

From (1.4), one may compute the corresponding top-hat quantities of velocity, density and radius:

$$
W_{T}=\frac{M}{G} \quad \frac{\rho_{T}}{\rho_{a}}=\frac{1}{1+\frac{\rho_{a} B}{g G}} \quad b_{T}=\sqrt{\frac{1}{\pi} \frac{G^{2}}{\rho_{T} M}}
$$

leading to

$$
\begin{aligned}
W_{T} & =\left(\frac{\rho_{a} B^{2}}{\Gamma_{0}^{2} G_{s}}\right)^{1 / 5} \frac{\left[\left(\frac{3}{8} \mathcal{Z}+2^{3 / 8}\right)^{8 / 3}-2\right]^{1 / 2}}{\left(\frac{3}{8} \mathcal{Z}+2^{3 / 8}\right)^{5 / 3}} \\
\frac{\rho_{T}}{\rho_{a}} & =\frac{1}{1+\frac{1-\eta_{s}}{\eta_{s}}\left(\frac{3}{8 \cdot 2^{3 / 8}} \mathcal{Z}+1\right)^{-5 / 3}} \\
b_{T} & =\frac{1}{\sqrt{\pi}}\left(\frac{3}{8}\right)^{2 / 3} \Gamma_{0} L_{0} \frac{\left(\frac{3}{8 \cdot 2^{3 / 8}} \mathcal{Z}+1\right)^{5 / 3}\left[1+\frac{1-\eta_{s}}{\eta_{s}}\left(\frac{3}{8 \cdot 2^{3 / 8}} \mathcal{Z}+1\right)^{-5 / 3}\right]^{1 / 2}}{\left[\left(\frac{3}{8 \cdot 2^{3 / 8}} \mathcal{Z}+1\right)^{8 / 3}-1\right]^{1 / 4}} .
\end{aligned}
$$

For the computation of density, we used (3.15) to express $G_{s}$ as a function of the density ratio at the source $\eta_{s}$. Figures $1-3$ give the evolution of these three quantities with $\mathcal{Z}$. Note that the evolutions of $\rho_{T} / \rho_{a}$ and $b_{T}$ with non-dimensional height $\mathcal{Z}=z / L$ depend on the density ratio at the source $\eta_{s}$, whereas $W_{T}$ is independent of this quantity, giving a clear confirmation that $L$ is a relevant characteristic length for height.

Combining (3.12) and (3.13) with the definition of the Richardson number (1.8) and 
the expression for $\alpha,(2.7)$, we get

$$
\frac{\alpha}{\alpha_{0}}=\left(\frac{\left(3 \mathcal{Z} / 8+2^{3 / 8}\right)^{8 / 3}}{\left(3 \mathcal{Z} / 8+2^{3 / 8}\right)^{8 / 3}-2}\right)^{1 / 4} .
$$

Figure 4 shows the evolution of $\alpha / \alpha_{0}$ with height, which is qualitatively similar to the experimental curve of Pham et al. (2005) (their figure 16). Unfortunately, a quantitative comparison is not possible because the value of $B$ is unknown in Pham et al. (2005).

Figure 1 shows that for small $\mathcal{Z}=z / L$ the plume acceleration is strong and that $W_{T} / W_{\text {ref }}$ reaches a local maximum, here within (approx.) 2.5 characteristic length scales of the source. Subsequently, the plume fluid decelerates gradually to follow the pureplume asymptotic trend of $W_{T} / W_{\text {ref }}=(8 / 3)^{1 / 3} \mathcal{Z}^{1 / 3}$ (dashed line) at large $\mathcal{Z}$.

Evidently, dilution of the plume within the near-field region of strong acceleration is also strong (e.g. figure 2 for $\mathcal{Z}=z / L \lesssim 2.5$ ), moreover the rate of dilution decreases with increasing density ratio $\eta_{s}$. Further above the source, the rate of dilution slows and, at a given height, the weakest dilution is achieved with the largest density ratio.

Consistent with the acceleration and dilution of the plume (figures 1 and 2), figure 3 shows that the plume contracts for small $\mathcal{Z}$, before expanding at greater heights. In all cases a pronounced narrowing of the plume is predicted; the height above the source that corresponds to the plume neck (its narrowest width) is reduced as the density ratio increases and the plume neck is narrower. Notably, the vertical non-dimensional height corresponding to the narrowest width depends on the value of density at the source $\eta_{s}$, while the height at which the plume velocity reaches a local maximum (figure 1) does not depend on it. For highly non-Boussinesq plumes (typically $\eta_{s} \lesssim 0.3$ ), the neck is higher than the maximal velocity height, whereas it is below this height for $\eta_{s} \gtrsim 0.3$. This prediction of the model is rather subtle but can be understood physically on account of the reduction in entrainment experienced by highly non-Boussinesq plumes (the $\sqrt{\rho_{T} / \rho_{a}}$ term in (1.9)). With this in mind, if one considers two plumes, one of which is highly non-Boussinesq, with identical source fluxes of buoyancy and mass (and hence each with the same acceleration length $L$ ), whilst they reach their maximum velocity at the same height as it is set by the value of $L$, the height required to attain the local state of balance corresponding to the neck is greater for the highly non-Boussinesq plume as a consequence of the aforementioned reduced entrainment. Specifically, reduced entrainment results in a slower dynamical variability in the plume with height, e.g. due to slower vertical variation in mass flux.

\subsection{Effect of varying $\omega$}

In the general case, the function $\Phi_{\omega}(\cdot)$ has to be computed numerically. It is fruitful to compare plume solutions with different $\omega$ for a given source condition. Following Hunt \& van den Bremer (2010) and also Candelier \& Vauquelin (2012), to perform this comparison we concentrate on diagrams of $\Gamma / \Gamma_{0}$ as a function of height, since $G(z)$ and $M(z)$ may be reconstructed from knowledge of $B, G_{s}$ and $\Gamma / \Gamma_{0}$ at any height, from (2.11), which becomes, after some algebra

$$
G=G_{s}\left[1-\left(\frac{\Gamma_{0}}{\Gamma}\right)^{1-\omega}\right]^{\frac{-1}{2(1-\omega)}}
$$

and $M$ as a function of $\Gamma$ follows from (1.8) and the above expression for $G$.

However, the scaling used so far for height depends on $\omega$. Therefore, to perform a comparison which is straightforward to interpret, we rescale the height so as to be independent of $\omega$. To this end, we take the acceleration length for the reference entrainment 


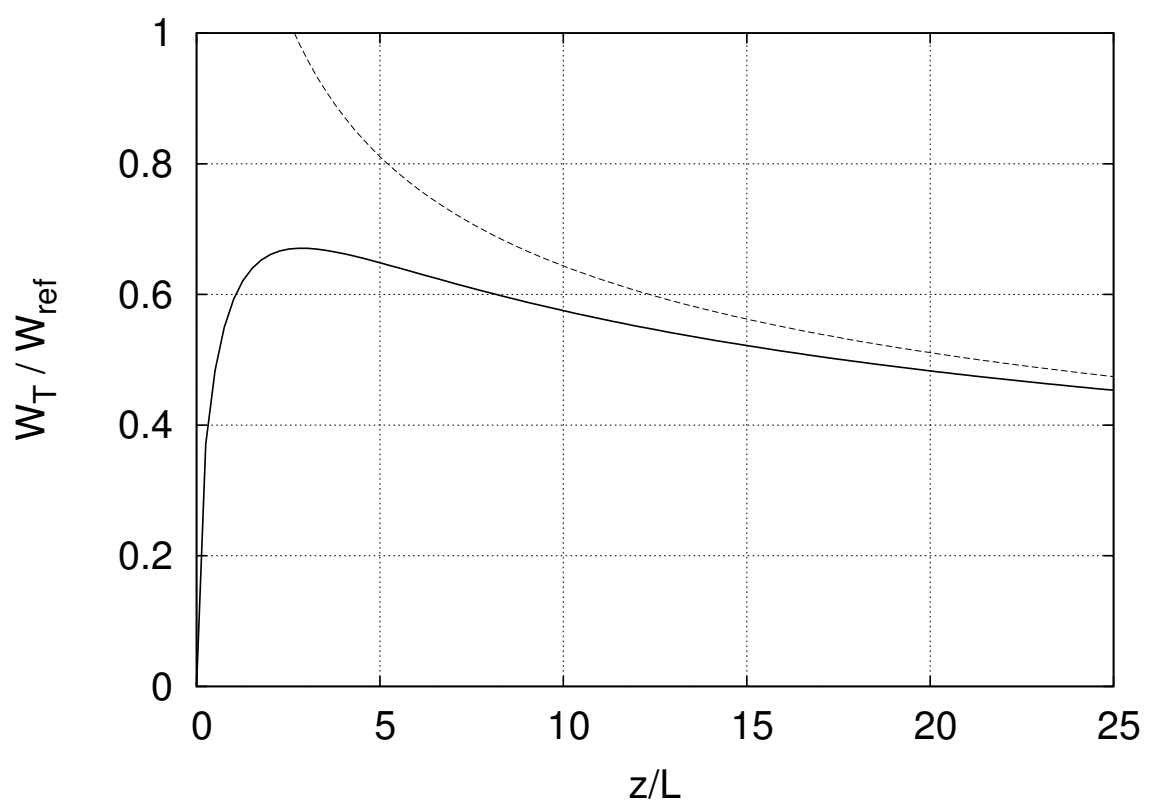

Figure 1. Normalised plume vertical velocity $W_{T} / W_{\text {ref }}$ as a function of normalised distance from the source $\mathcal{Z}=z / L$ for $\omega=1 / 5$, with $W_{\text {ref }}=\left[\left(\rho_{a} B^{2}\right) /\left(\Gamma_{0}^{2} G_{s}\right)\right]^{1 / 5}$. The dashed line shows the large $\mathcal{Z}$ pure-plume asymptotics $(8 / 3)^{1 / 3} \mathcal{Z}^{1 / 3}$.

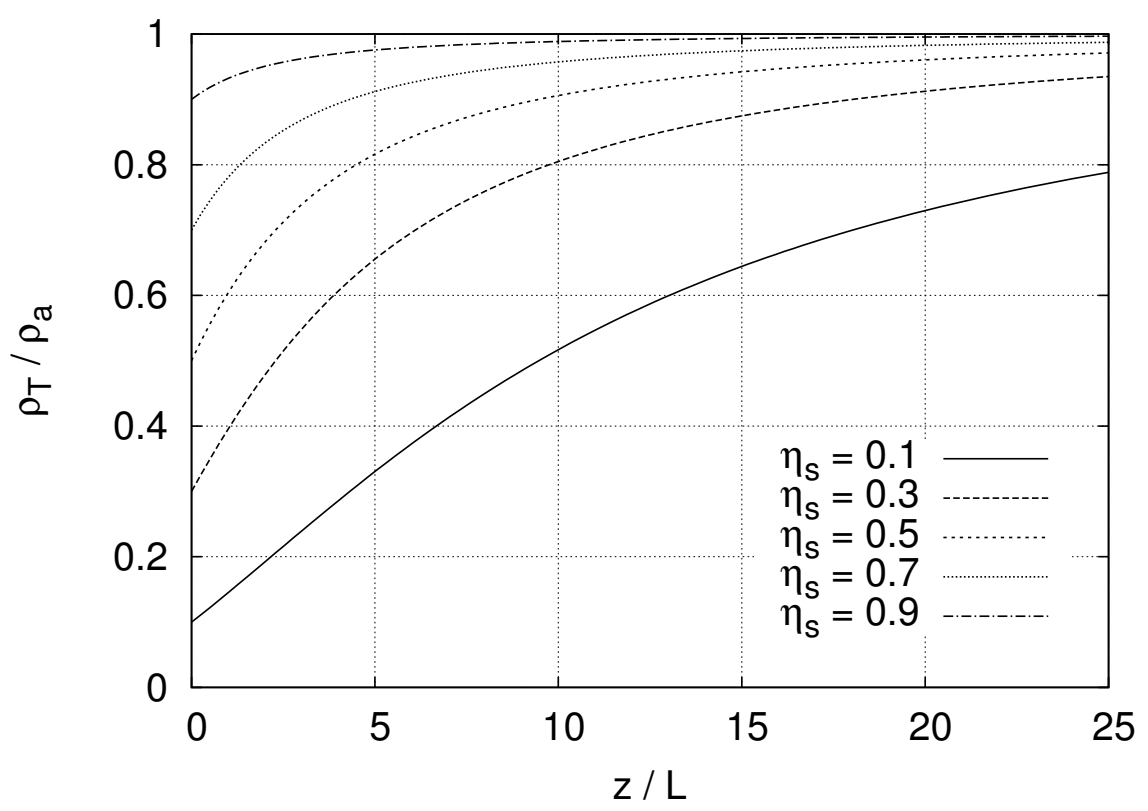

FiguRE 2. Normalised plume density $\rho_{T} / \rho_{a}$ as a function of distance from source $\mathcal{Z}=z / L$ for $\omega=1 / 5$ and source density ratios of $\eta_{s}=\{0.1,0.3,0.5,0.7$ and 0.9$\}$. 


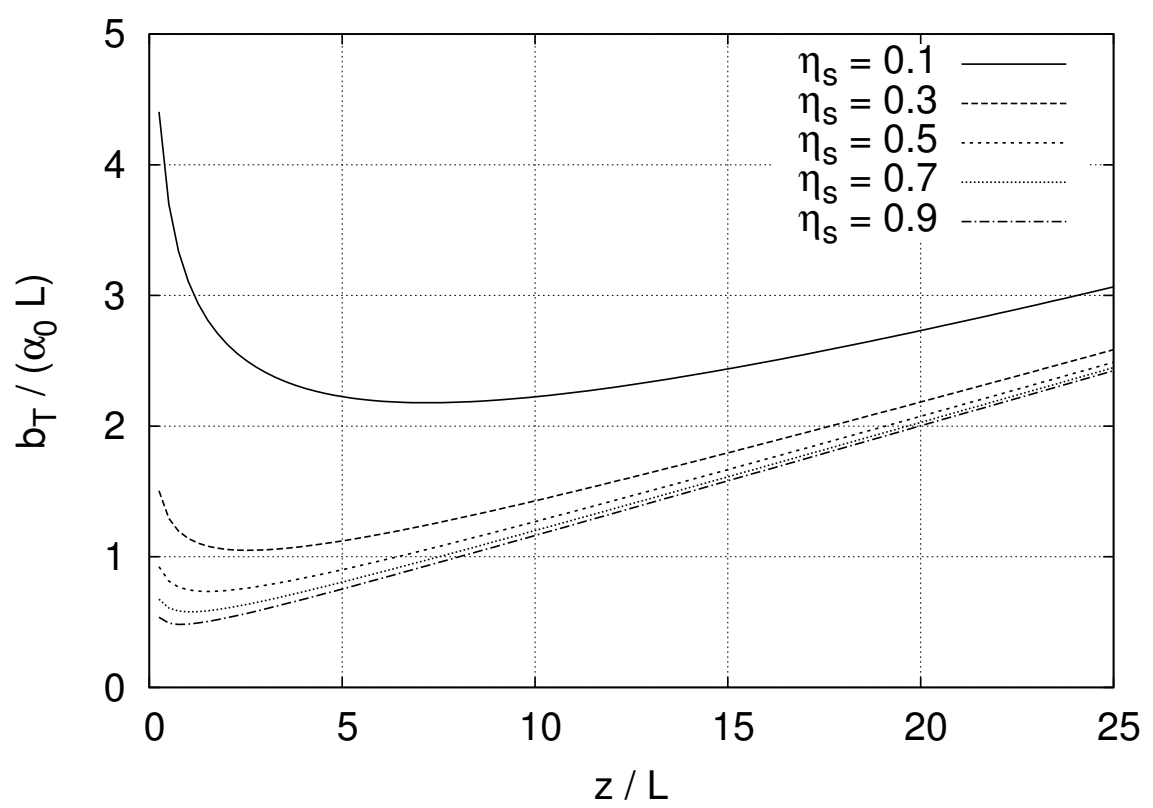

Figure 3. Normalised plume width $b_{T} /\left(\alpha_{0} L\right)$ as a function of distance from source $\mathcal{Z}=z / L$ for $\omega=1 / 5$ and source density ratios of $\eta_{s}=\{0.1,0.3,0.5,0.7$ and 0.9$\}$.

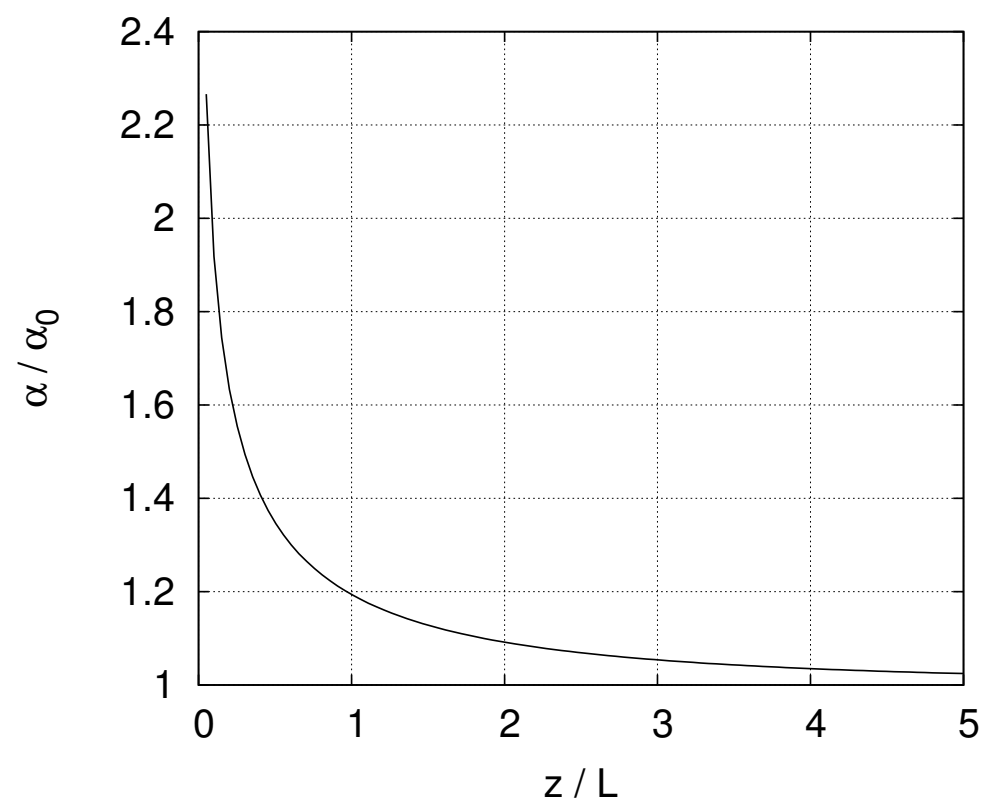

FiguRE 4. Variation of the entrainment coefficient $\alpha$ divided by its pure-plume value $\alpha_{0}$ as a function of the normalised distance from the source $\mathcal{Z}=z / L$ for $\omega=1 / 5$. 


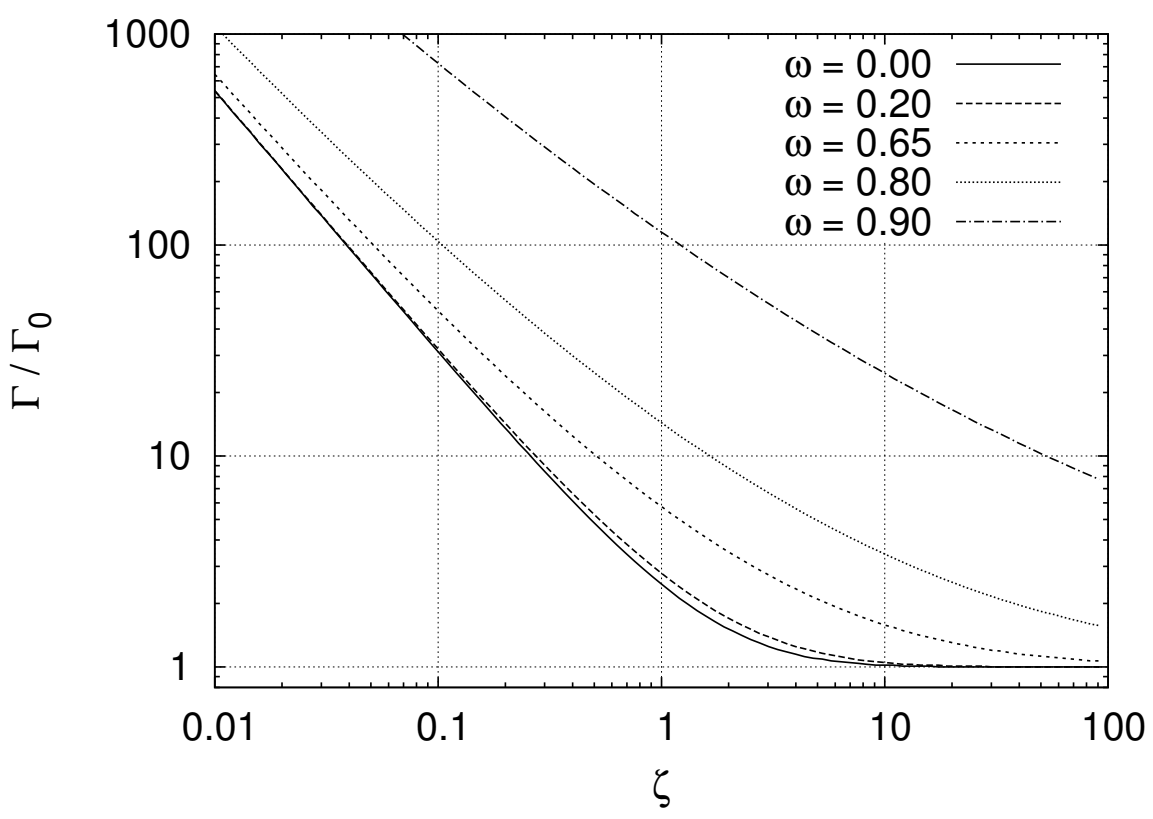

Figure 5. Plume Richardson number normalised by its pure-plume value $\Gamma / \Gamma_{0}$ as a function of non-dimensional distance to the source $\zeta=z / L_{0}$.

coefficient $\alpha_{0}$ as

$$
L_{0}=\frac{2^{\frac{7}{10}}}{5 \Gamma_{0}^{4 / 5}} \frac{G_{s}^{3 / 5}}{\rho_{a}^{3 / 5} B^{1 / 5}}
$$

and define

$$
\zeta=\frac{z}{L_{0}}=\frac{L_{0}}{L} \cdot \mathcal{Z}
$$

We have

$$
\frac{\Gamma}{\Gamma_{0}}=\frac{1}{\Gamma_{0}} \rho_{a}^{1 / 2} \frac{B G^{2}}{M^{5 / 2}}
$$

and, from (3.12) and (3.13), after some algebra:

$$
\frac{\Gamma}{\Gamma_{0}}=\left(\frac{\Phi_{\omega}(\mathcal{Z})+1}{\Phi_{\omega}(\mathcal{Z})-1}\right)^{1 /(1-\omega)}=\left(\frac{\Phi_{\omega}\left(\left(L_{0} / L\right) \zeta\right)+1}{\Phi_{\omega}\left(\left(L_{0} / L\right) \zeta\right)-1}\right)^{1 /(1-\omega)} .
$$

Figure 5 shows the influence of the value of the exponent $\omega$ on the variation of $\Gamma / \Gamma_{0}$ with height $\zeta$. It is seen that for small $\omega$, the curves are very close to each other; the difference between the cases $\omega=0$ and $\omega=1 / 5$ is barely visible. This close similarity indicates that the bulk local plume dynamic, as captured by $\Gamma(\zeta)$, is relatively insensitive to changes in entrainment caused by these variations in $\omega$. The fact that the model with $\omega=1 / 5$ is purely algebraic, whilst yielding results very close to the constant entrainment model (the latter, by contrast, requiring tedious calculations, cf. Carlotti \& Hunt 2005), may be regarded as good news for enabling the simple and rapid application of plume models. On the other hand, for larger $\omega$, the curves tend to separate more and more for a given incremental change in $\omega$, and thus the plume behaviour is sensitive to $\omega$. 


\section{Comparisons with the available lazy plume data}

\subsection{Lazy turbulent plumes issuing from injection nozzles}

So far, the model for a plume with variable entrainment was used over its complete range of validity, from the origin (where $M=M_{s}=0$ and $G=G_{s}$ ) to infinite height. Moreover, at the source, as may be seen from our previous considerations, the density ratio is given by (3.15) and the top-hat velocity is zero (figure 1), the top-hat radius is infinite (figure 3 ). This is not an effect of the choice of normalisation, but comes from the definition of the top-hat radius in (1.4) and the asymptotic behaviour of the fluxes. However, in practical cases, these theoretical source conditions are never met. Depending on the context or application, plumes may rise from a heated plate, a combustion source, or a nozzle discharging fluid at a given rate and temperature. Obtaining a description of the source conditions at a heated plate, or at a combustion source, would require the application of specific models (heat transfer at the plate, or combustion), and we therefore decide to concentrate on plumes produced by fluid issuing turbulently from a nozzle. We consider a circular nozzle of radius $b_{n}$, located at a height $z=0$. It is assumed that the design of the nozzle is such that the flow is uniform across the nozzle exit, with density $\rho_{n}$ (we define $\eta_{n}=\rho_{n} / \rho_{a}$ ) and vertical discharge velocity $W_{n}$. The fluxes at the nozzle are

$$
B_{n}=\pi g\left(1-\eta_{n}\right) W_{n} b_{n}^{2} \quad M_{n}=\pi \rho_{n} W_{n}^{2} b_{n}^{2} \quad G_{n}=\pi \rho_{n} W_{n} b_{n}^{2},
$$

and therefore all parameters at the nozzle may be written as functions of $W_{n}, b_{n}$ and $\eta_{n}$ :

$$
\begin{aligned}
\frac{\Gamma_{n}}{\Gamma_{0}} & =\frac{5}{8 \alpha_{0}} \frac{1-\eta_{n}}{\eta_{n}^{1 / 2}} \frac{g b_{n}}{W_{n}^{2}} \\
J_{n} & =\frac{1}{2}\left(\pi \eta_{n} \rho_{a} W_{n} b_{n}^{2}\right)^{2(1-\omega)}\left[1-\left(\frac{\Gamma_{0}}{\Gamma_{n}}\right)^{1-\omega}\right] \\
I_{n} & =\frac{1}{2}\left(\pi \eta_{n} \rho_{a} W_{n} b_{n}^{2}\right)^{2(1-\omega)}\left[1+\left(\frac{\Gamma_{0}}{\Gamma_{n}}\right)^{1-\omega}\right] \\
L & =\frac{1}{2^{4 / 5}(1-\omega) \alpha_{0}}\left(\frac{\Gamma_{0}}{\Gamma_{n}}\right)^{1 / 5}\left[\frac{1}{2}\left(1-\left(\frac{\Gamma_{0}}{\Gamma_{n}}\right)^{1-\omega}\right)\right]^{\frac{3}{10(1-\omega)}} \eta_{n}^{1 / 2} b_{n} .
\end{aligned}
$$

From (3.5), we have

$$
G_{s}=2^{\frac{1}{2(1-\omega)}} J_{n}^{\frac{1}{2(1-\omega)}}=\pi \rho_{n} W_{n} b_{n}^{2}\left[1-\left(\frac{\Gamma_{0}}{\Gamma_{n}}\right)^{1-\omega}\right]^{\frac{1}{2(1-\omega)}} .
$$

We define the virtual origin as the location of a source $\left(M_{s}=0, G_{s}\right)$ needed to create the desired conditions at the nozzle. If the height of the virtual origin is written $z_{v}$, and defining $\mathcal{Z}_{v}=z_{v} / L$, from (3.12), we have

$$
G_{n}=G_{s} \cdot\left(\frac{\Phi_{\omega}\left(\mathcal{Z}_{v}\right)+1}{2}\right)^{1 /(2(1-\omega))}
$$

leading to

$$
\mathcal{Z}_{v}=\hat{\Phi}_{\omega}\left(2\left(\frac{G_{n}}{G_{s}}\right)^{2(1-\omega)}-1\right)=\hat{\Phi}_{\omega}\left(\frac{1+\left(\Gamma_{0} / \Gamma_{n}\right)^{1-\omega}}{1-\left(\Gamma_{0} / \Gamma_{n}\right)^{1-\omega}}\right) .
$$

Now, using (3.12) and (3.13), the evolutions of $G$ and $M$ are given by

$$
\begin{aligned}
& G=G_{n} \cdot\left(\frac{\Phi_{\omega}\left(\mathcal{Z}+\mathcal{Z}_{v}\right)+1}{\Phi_{\omega}\left(\mathcal{Z}_{v}\right)+1}\right)^{1 /(2(1-\omega))} \\
& M=\left(\frac{\sqrt{\rho_{a}} B}{\Gamma_{0}}\right)^{2 / 5} G_{n}^{4 / 5} \cdot\left(\frac{\Phi_{\omega}\left(\mathcal{Z}+\mathcal{Z}_{v}\right)-1}{\Phi_{\omega}\left(\mathcal{Z}_{v}\right)+1}\right)^{2 /(5(1-\omega))} .
\end{aligned}
$$




\subsection{Evolution of mass flow with height}

We introduce the so-called source flow rate length (Kaye \& Hunt, 2009)

$$
L_{q}=\frac{G_{n}}{\sqrt{\rho_{n} M_{n}}} .
$$

Using (3.7) and (4.7), after some algebra, we may express $L / L_{q}$ as a function of the density ratio $\rho_{n} / \rho_{a}$ and $\Gamma_{n} / \Gamma_{0}$ :

$$
\frac{L}{L_{q}}=\frac{2^{7 / 10}}{5 \Gamma_{0}} \cdot \frac{2^{\frac{-3 \omega}{10(1-\omega)}}}{1-\omega} \cdot \sqrt{\frac{\rho_{n}}{\rho_{a}}} \cdot\left[1-\left(\frac{\Gamma_{0}}{\Gamma_{n}}\right)^{1-\omega}\right]^{\frac{-3}{10(1-\omega)}} \cdot\left(\frac{\Gamma_{0}}{\Gamma_{n}}\right)^{1 / 5} .
$$

We define

$$
\hat{z}=\frac{z}{L_{q}}=\mathcal{Z} \cdot \frac{L}{L_{q}} .
$$

Therefore, with the single source parameter $\Gamma_{n} / \Gamma_{0}$, one may compute $\mathcal{Z}_{v}$ from (4.8) and $G / G_{n}$ as a function of $\hat{z}$ :

$$
\frac{G}{G_{n}}=\left(\frac{\Phi_{\omega}\left(\frac{L_{q}}{L} \hat{z}+\mathcal{Z}_{v}\right)+1}{\Phi_{\omega}\left(\mathcal{Z}_{v}\right)+1}\right)^{\frac{1}{2(1-\omega)}} .
$$

For very lazy plumes, $\left(\Gamma_{0} / \Gamma_{n}\right)^{1-\omega} \ll 1$. As a consequence, from $(4.12), L / L_{q} \propto$ $\left(\Gamma_{0} / \Gamma_{n}\right)^{1 / 5}$, and thus $\left(L_{q} / L\right) \hat{z} \propto\left(\Gamma_{n} / \Gamma_{0}\right)^{1 / 5} \hat{z}$. Therefore, according to this model, the curves of $G / G_{n}$ plotted as a function of $\left(\Gamma_{n} / \Gamma_{0}\right)^{1 / 5} \cdot \hat{z}$ will coincide.

\subsection{Comparison of our model with experiments}

An experimental study of very lazy Boussinesq plumes, based on saline releases $(\mathrm{NaCl})$ in fresh water, was performed by Kaye \& Hunt (2009). Based on their measurements, they plot $\rho_{n} G / \rho_{T} G_{n}$ as a function of $\hat{z}$ for four plumes $\Gamma_{n} / \Gamma_{0}=\left\{9.1 \cdot 10^{4}, 1.6 \cdot 10^{5}, 5.4 \cdot 10^{5}\right.$, $5.7 \cdot 10^{6}$ and $\left.5.4 \cdot 10^{7}\right\}$. Note that since, in their case, the flow fulfils the Boussinesq approximation, $\rho_{T}=\rho_{n}=\rho_{a}$ to a good precision and, therefore, $\rho_{n} G / \rho_{T} G_{n}=G / G_{n}$.

The first step towards a comparison of our model with experiment is to choose appropriate values for the two parameters of the model, namely for $\alpha_{0}$ and $\omega$. Here we choose to fix, rather arbitrarily, the pure-plume constant entrainment coefficient as $\alpha_{0}=0.1$ and to determine a value of $\omega$ that gives a good comparison with the measurements of Kaye \& Hunt (2009). For $\Gamma_{n} / \Gamma_{0}=5.4 \cdot 10^{5}$ we find $\omega=0.62$ (see figure 6 ). As an aside, we find that the value of $\omega$ is relatively insensitive to the choice of $\alpha_{0}$, with $\omega$ decreasing marginally to $\omega=0.61$ with $\alpha_{0}=0.118$.

Having chosen these values for $\alpha_{0}$ and $\omega$, we compare the predictions of the model with the Kaye \& Hunt (2009) data for other values of $\Gamma_{n} / \Gamma_{0}$. These comparisons are shown in figure 7 . In spite of the simplistic character of the assumptions made regarding the entrainment with (2.7), surprisingly good agreement is shown between the model predictions and the available experimental data. It should be noted that a constant entrainment solution would not lead at all to the same result, even in terms of order of magnitude - indeed, the result would not be visible on the figure.

Finally, to highlight the implications of the scalings arrived at in $(5.12)-(5.14)$, in figure 8 we replot the scaled mass flux data $G / G_{n}$, now as a function of $\hat{z} \cdot\left(\Gamma_{n} / \Gamma_{0}\right)^{1 / 5}$. Evidently, this scaling collapses the data well, particularly for small $\hat{z} \cdot\left(\Gamma_{n} / \Gamma_{0}\right)^{1 / 5}$. Kaye \& Hunt (2009) scale their data on the 1/3rd power, and although this resulted in a reasonable collapse of their data, they were not able to offer an argument therein for this choice of exponent. 


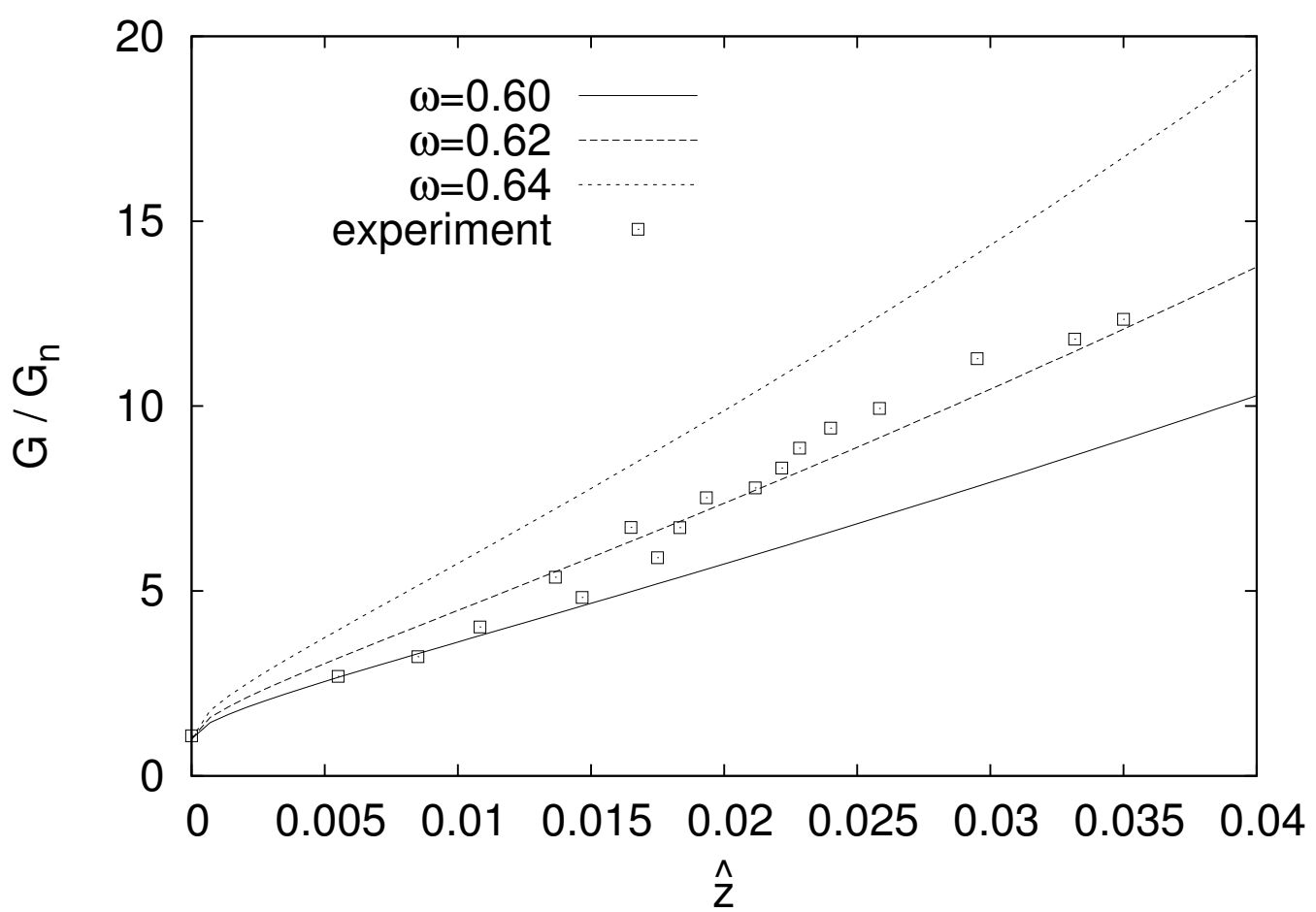

Figure 6 . Evolution of $G / G_{n}$ as a function of $\hat{z}=z / L_{q}$ predicted by the present model with $\Gamma_{n} / \Gamma_{0}=5.4 \cdot 10^{5}$ for $\omega=\{0.60,0.62$ and 0.64$\}$, compared with the experimental results of Kaye \& Hunt (2009).

\section{Conclusion}

The present paper focuses on variable entrainment lazy plumes. Section 2 discusses the possibility of entrainment varying with local Richardson number in the plume. After a rapid review of existing formulations, a power-law variation was used, with an empirical coefficient $\omega$, which may have a value between 0 and 1 ( 1 being excluded). Taking $\omega=0$ corresponds to the usual model, wherein the entrainment does not depend on the Richardson number, while $\omega=0.36$ corresponds to the variation of entrainment suggested by several references in the literature based on weakly lazy plumes. For a pure plume, the model is independent of the value of $\omega$, being always identical to the MTT model.

The plume equations were subsequently solved for any $\omega$, for pure and lazy plumes, and the solutions then analysed in more detail (§3). It is shown, in particular, that the cases $\omega=0$ and $\omega=1 / 5$ lead to very similar quantitative results, while the case $\omega=1 / 5$ may be computed easily with usual functions (especially polynomials) only. This advocates the idea of selecting systematically $\omega=1 / 5$ for cases where the effect of variation of entrainment is weak, instead of $\omega=0$ which leads to tedious calculations.

For very lazy plumes, for which it is known that a constant entrainment model is not at all appropriate, we concentrate $(\S 4)$ on plumes that develop when fluid is released from turbulent injection nozzles. We compare the outcome of the present model with experimental data from Kaye \& Hunt (2009). Good agreement is shown with $\omega=0.62$ and, to our knowledge, this is the first model to give such good agreement with experiments for 


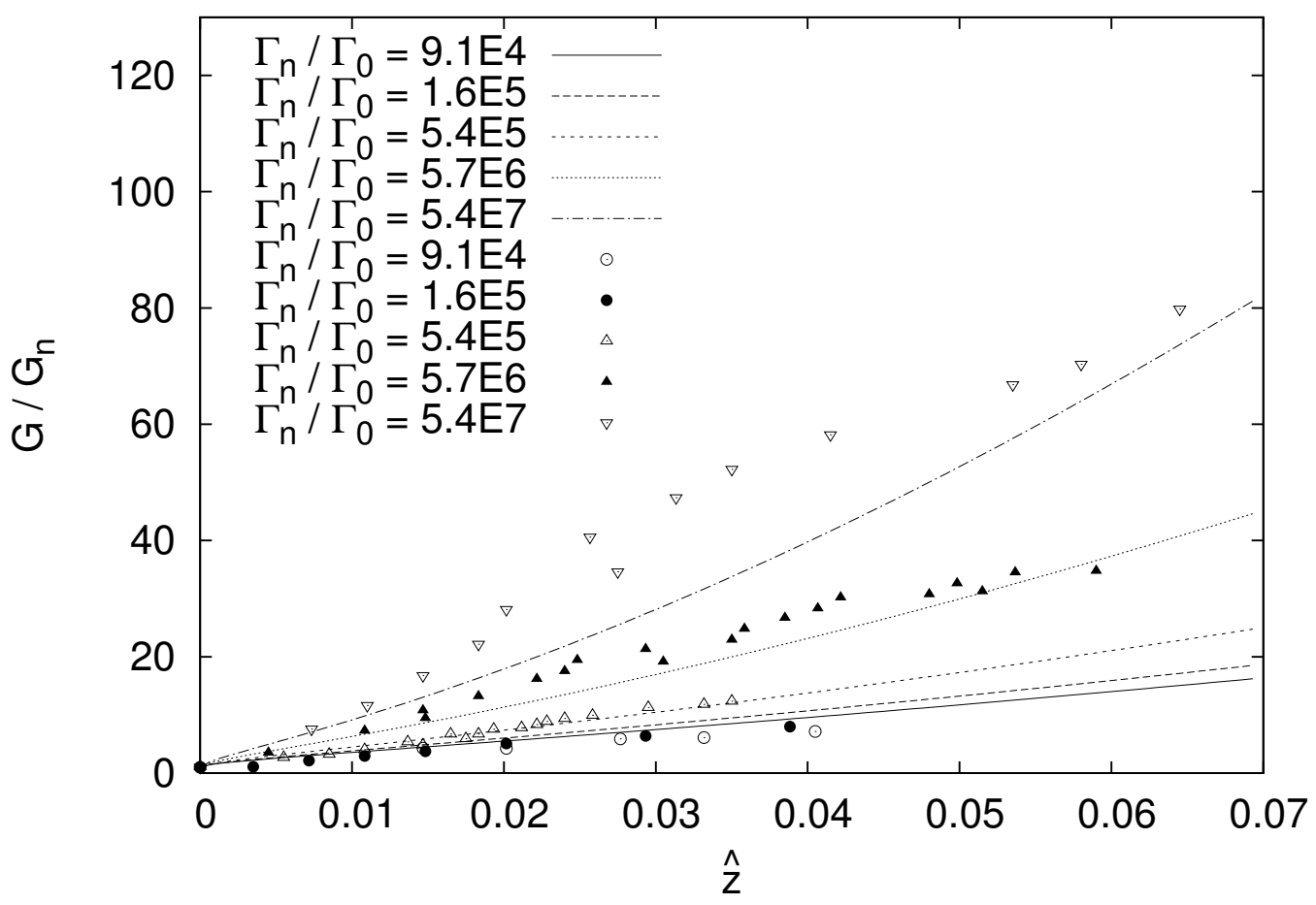

Figure 7. Evolution of $G / G_{n}$ as a function of $\hat{z}$ : experimental results of Kaye \& Hunt (2009) and prediction from the present model $\alpha_{0}=0.1$ and $\omega=0.62$.

very lazy plumes. It shows that the concept of a simple entrainment model is good, even in cases far from the initial assumptions underlying MTT. A next step in this study will be to compare the results of the model with other very lazy plumes in order to determine whether the value of $\omega$ is comparable across all plume experiments, which would mean that its dependence on the precise conditions of release of the fluid is weak. Evidently, this step will first require additional measurements to be made on lazy plumes.

\section{Acknowledgements}

The authors would like to thank R.J. Perkins, P. Salizzoni and D. Lamalle for fruitful discussions, and anonymous referees of a previous version of this paper.

\section{REFERENCES}

Carlotti, P. 2013. Eléments de mécanique des fluides pour la modélisation des incendies. CSTB research report, http://hal-cstb.archives-ouvertes.fr/CSTB/tel-00978098/fr/

Carlotti, P. \& Hunt, G.R. 2005. Analytical solutions for non-Boussinesq plumes. J. Fluid Mech. 538:343-359.

Candelier, F. \& Vauquelin, O. 2012. Matched asymptotic solutions for turbulent plumes. J. Fluid Mech. 699:489-499.

Caulfield, C.P. 1991 Stratification and buoyancy in geophysical flows. Ph.D. thesis, DAMTP, University of Cambridge, UK.

Ezzamel, A., Salizzoni, P. \& Hunt, G.R. 2015. Dynamical variability of axisymmetric buoyant plumes. J. Fluid Mech. 765:576-611. 


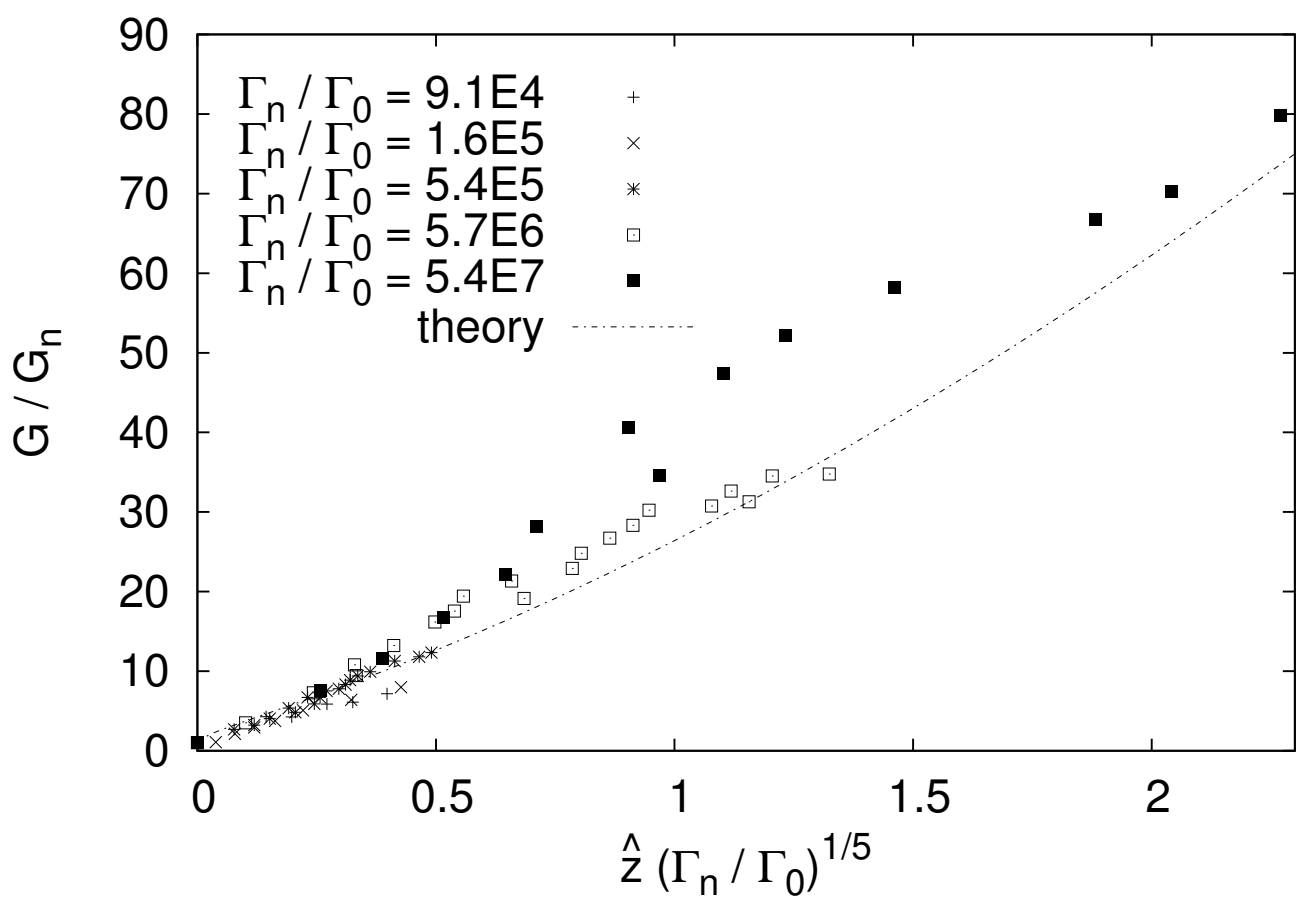

FIGURE 8 . Evolution of $G / G_{n}$ as a function of $\hat{z} \cdot\left(\Gamma_{n} / \Gamma_{0}\right)^{1 / 5}$ predicted by the present model for $\omega=0.62$, compared with the experimental results of Kaye \& Hunt (2009); with this scaling an approximate collapse of the data is evident, notably for small $\hat{z} \cdot\left(\Gamma_{n} / \Gamma_{0}\right)^{1 / 5}$.

Fannelop, T.K. \& Webber, D.M. 2003 On buoyant plumes rising from area sources in a calm environment J. Fluid Mech. 497: 319-334.

Fischer, H.B., List, E.J., Koy, R.C-Y., Imberger, J. \& Brooks, N.H. 1979. Mixing in inland and coastal waters. Academic Press. 483 pp.

Hunt, G.R. \& Kaye, N.G. 2001. Virtual origin correction for lazy turbulent plumes. J. Fluid Mech. 435:377-396.

Hunt, G.R. \& van den Bremer, T.S. 2010. Classical plume theory: 1937-2010 and beyond. IMA Journal of Applied Mathematics, 76:1-25.

Kaye, N.B. 2008 Turbulent plumes in stratified environments: A review of recent work. Atmosphere-Ocean 46:433-441.

Kaye, N.B. \& Hunt, G.R. 2009. An experimental study of large area source turbulent plumes. Int. J. of Heat and Fluid Flow 30:1099-1105.

Lamalle, D., Carlotti, P., Salizzoni, P. \& Perkins, R.J. 2013. Simulations aux grandes échelles de panaches. 21st Congrès Français de Mécanique, Bordeaux, France, edited by I. Iordanoff, Association Française de Mécanique, http://documents.irevues.inist.fr/handle/2042/52776.

List E.J. 1982. Mechanics of turbulent buoyant jets and plumes. Turbulent Buoyant Jets and Plumes, ed. W. Rodi, pp. 1-68, Pergamon.

Morton, B.R. 1959. Forced plumes. J. Fluid Mech. 5:151-163.

Morton, B.R., Taylor, G. \& Turner, J.S. 1956. Turbulent gravitational convection from maintained and instantaneous sources. Proc. Roy. Soc. A 23:1-23.

Pham, M.V., Plourde, F. \& Kim, S.D. 2005. Three-dimensional characterisation of a pure thermal plume. Transactions of the ASME 127:624-636. 
Priestley, C.H.B. \& Ball, F.K. 1955. Continuous convection from an isolated source of heat. $Q$. J. R. Met. Soc. 81:144-157.

Rooney, G.G. \& Linden, P.F. 1996. Similarity considerations for non-Boussinesq plumes in an unstratified environment. J. Fluid Mech. 318:237-250.

Taylor, G.I. 1958. Flow induced by jets. J. of Aero/Space Sciences 25:464-465.

van Reeuwijk, M. \& Craske, J. 2015. Energy-consistent entrainment relations for jets and plumes. J. Fluid Mech. 782:333-355.

van Reeuwijk, M., Salizzoni, P., Hunt, G.R., and Craske, J. 2016. Turbulent transport and entrainment in jets and plumes: a DNS study. arXiv preprint, http://arxiv.org/abs/1603.09078

Wang, H.W. \& Law, A.W.K. 2002. Second order integral model for a round turbulent buoyant jet. J. Fluid Mech. 459:397-428.

Zeldovich, Y. B. 1937. The asymptotic laws of freely-ascending convective flows. Zh. Eksp. Teor. Fiz. 7: 1463-1465 (in Russian). English translation in Selected Works of Yakov Borisovich Zeldovich, 1, 1992 (J. P. Ostriker ed.) 82-85. Princeton University Press.

\section{Appendix A. Taking into account variable specific heat in hot gaseous plumes}

For a hot gaseous plume, the buoyancy equation is obtained using enthalpy and mass conservation (see, for example, Carlotti \& Hunt 2005). When it is assumed that specific heat is constant $C_{P}(T)=C_{P}^{0}$, so that enthalpy is $H(T)=H\left(T_{\text {ref }}\right)+C_{P}^{0}\left(T-T_{\text {ref }}\right)$ ( $T_{\text {ref }}$ being a reference temperature), then the buoyancy flux $B$ as defined by (1.1) is independent of height in an unstratified environment.

However, in the general case, $C_{P}$ is not constant, $C_{P}=(\partial H / \partial T) \mid P$, which in a context of low Mach flow with constant thermodynamic pressure (see for example Carlotti 2013), leads to $H(T)=H\left(T_{\text {ref }}\right)+\int_{T_{r e f}}^{T} C_{P}\left(T^{\prime}\right) d T^{\prime}$. Defining

$$
C_{P}^{\prime}=\int_{T_{\text {ref }}}^{T} \frac{C_{P}\left(T^{\prime}\right)}{T-T_{\text {ref }}} d T^{\prime}
$$

we have for variable specific heat $H(T)=H\left(T_{\text {ref }}\right)+C_{P}^{\prime}(T) \times\left(T-T_{\text {ref }}\right)$. In the low Mach number formulation, one may combine the general tridimensional enthalpy and mass conservation equations to obtain an equation where the time derivative terms cancel (Carlotti 2013):

$$
\left(1+\frac{T}{C_{P}^{\prime}} \frac{d C_{P}^{\prime}}{d T}\right) \nabla \cdot \mathbf{u}=\frac{1}{C_{P}^{\prime} \rho_{a} T_{a}}\left[\nabla \cdot\left(\rho C_{P}^{\prime} \kappa \nabla T\right)+\mathcal{S}\right],
$$

where $\mathbf{u}$ is the velocity field, $\kappa$ the diffusivity and $\mathcal{S}$ represents sources and sinks of heat. Note that this combination of mass and enthalpy conservation may be interpreted as a justification of the so-called volume conservation in the present context.

Defining

$$
\mathbf{v}=\frac{C_{P}^{\prime} \rho_{a}-C_{P}^{0} \rho}{C_{P}^{0} \rho_{a}} \mathbf{u}
$$

(with $C_{P}^{0}=C_{P}\left(T_{r e f}\right)$ ), the previous equation may be written, using the ideal gas law and after some algebra

$$
\nabla \cdot \mathbf{v}=\frac{1}{C_{P}^{\prime} \rho_{a} T_{a}}\left[\nabla \cdot\left(\rho C_{P}^{\prime} \kappa \nabla T\right)+\mathcal{S}\right] .
$$

Then, integrating on a cylinder of diameter $R$ ( $R$ larger than the plume radius) centred on the vertical axis starting at the source, between heights $z$ and $z+d z$, and using the 
divergence theorem, $\iiint_{\Omega} \nabla \cdot \mathbf{v} d V=\iint_{\partial \Omega} \mathbf{v} \cdot d \mathbf{S}$, where $\Omega$ is a closed control volume and $\partial \Omega$ is the boundary of this volume, one obtains in the limit $d z \rightarrow 0$

$$
\frac{d}{d z}\left(\iint_{r<R} g \frac{C_{P}^{\prime} \rho_{a}-C_{P}^{0} \rho}{C_{P}^{0} \rho_{a}} W d S\right)=\frac{2 \pi g}{C_{P}^{\prime} \rho_{a} T_{a}} \int_{0}^{R}\left[\nabla \cdot\left(\rho C_{P}^{\prime} \kappa \nabla T\right)+\mathcal{S}\right] r d r .
$$

As in the constant $C_{P}$ model, the diffusive terms on the right-hand side may be neglected (see MTT), and, taking the limit $R \rightarrow \infty$, one recovers, in the absence of sources

$$
\frac{d B^{\prime}}{d z}=0 \quad \text { where } \quad B^{\prime}(z)=\iint g \frac{C_{P}^{\prime} \rho_{a}-C_{P}^{0} \rho}{C_{P}^{0} \rho_{a}} W d S .
$$

As a consequence, provided this modified definition of buoyancy flux is taken, the conservation of buoyancy flux with height is still valid in gaseous plumes with specific heat varying with temperature. The effect of this correction for a $400^{\circ} \mathrm{C}$ air plume issuing from a nozzle into an ambient at room temperature was estimated through LES by Lamalle (2013, private communication) to be of order $10 \%$. Using the definition of classic buoyancy flux, some algebra leads to

$$
B-B^{\prime}=\iint g \frac{C_{P}^{0}-C_{P}^{\prime}}{C_{P}^{0}} W d S .
$$

Calculation of $C_{P}^{\prime}$ confirms a $10 \%$ order of magnitude for air and typical temperature between $20^{\circ} \mathrm{C}$ and $500^{\circ} \mathrm{C}$ (e.g. Carlotti (2013)). 